\title{
The Role of Molecular Testing and Enzyme Analysis in the Management of Hypomorphic Citrullinemia
}

\author{
David P. Dimmock ${ }^{1,{ }^{*},+}$, Pamela Trapane ${ }^{2}$, Annette Feigenbaum ${ }^{3}$, Catherine E. Keegan ${ }^{4}$, \\ Stephen Cederbaum ${ }^{5}$, James Gibson ${ }^{6}$, Michael J. Gambello ${ }^{7}$, Keith Vaux ${ }^{8}$, Patricia Ward ${ }^{1}$, \\ Gregory M. Rice ${ }^{9}$, Jon A Wolff ${ }^{9}$, William E. O'Brien ${ }^{1}$, and Ping Fang ${ }^{1}$ \\ ${ }^{1}$ Molecular and Human Genetics, Baylor College of Medicine, Houston, TX, USA \\ ${ }^{2}$ Genetics Center, Department of Pediatrics, Medical College of Wisconsin, Milwaukee, WI, USA \\ ${ }^{3}$ Division of Genetics, The Hospital for Sick Children, Toronto, ON, Canada \\ ${ }^{4}$ Dept. of Pediatrics, Division of Genetics, University of Michigan, Ann Arbor, MI, USA \\ ${ }^{5}$ Depts. of Psychiatry, Pediatrics and Human Genetics, UCLA, Los Angeles, CA, USA \\ ${ }^{6}$ University of Texas Health Science Center at San Antonio, San Antonio, TX; Now at Dell \\ Hospital for Children, Austin, TX, USA \\ 7 The University of Texas Medical School at Houston, Houston, TX, USA \\ ${ }^{8}$ Dept. of Pediatrics, University of California San Diego, San Diego, CA, USA \\ ${ }^{9}$ Division of Genetics and Metabolism, Department of Pediatrics, University of Wisconsin School \\ of Medicine and Public Health, Madison, USA
}

\section{Abstract}

Expanded newborn screening detects patients with modest elevations in citrulline; however it is currently unclear how to treat these patients and how to counsel their parents. In order to begin to address these issues, we compared the clinical, biochemical and molecular features of 10 patients with mildly elevated citrulline levels. Three patients presented with clinical illness whereas seven came to attention as a result of expanded newborn screening. One patient presented during pregnancy and responded promptly to IV sodium phenylacetate/sodium benzoate and arginine therapy with no long-term adverse effects on mother or fetus. Two children presented with neurocognitive dysfunction, one of these responded dramatically to dietary protein reduction. ASS enzyme activity was not deficient in all patients with biallelic mutations suggesting this test cannot exclude the ASS1 locus in patients with mildly elevated plasma citrulline. Conversely, all symptomatic patients who were tested had deficient activity. We describe four unreported mutations (p.Y291S, p.R272H, p.F72L and p.L88I), as well as the common p.W179R mutation. In Silico algorithms were inconsistent in predicting the pathogenicity of mutations. The cognitive benefit in one patient of protein restriction and the lack of adverse outcome in 7 others restricted from birth, suggest a role for protein restriction and continued monitoring to prevent neurocognitive dysfunction.

\footnotetext{
*Correspondence and reprint requests should be addressed to: David Dimmock, Genetics Clinic, Medical College of Wisconsin, 9000 West Wisconsin Avenue, Milwaukee, WI, 53226, Phone: (414) 266-3347 Fax: (414) 266-1616, Email: ddimmock@hmgc.mcw.edu. +Now at: Genetics Center, Department of Pediatrics, Medical College of Wisconsin, Milwaukee, WI, USA;

Conflict of Interest: None Declared
} 


\section{Keywords}

ASS1; Liver Failure; Drug Therapy; Pregnancy; DNA Diagnosis; Newborn Screening

\section{INTRODUCTION}

Argininosuccinate synthetase (ASS) catalyzes the third step in the urea cycle, the conversion of citrulline and aspartate into argininosuccinic acid with the cleavage of ATP into AMP and PPi. Complete deficiency of the enzyme results in classic citrullinemia. [McMurray et al., 1962, Beaudet et al., 1986; Su et al., 1982] This disorder is typically associated with elevations in citrulline above $1500 \mu \mathrm{M}$ and is associated with significant long term neurological disability [Bachmann 2003]. However it has been recognized that some patients with milder deficiency may present with later onset of learning disabilities, in acute crisis in pregnancy [Ruitenbeek et al.,2003] or as an incidental finding with good long term follow up [Wick at al 1975].

Expanded newborn screening for citrullinemia has been introduced in most states in the US over the past 15 years. In addition to classical ASS deficiency, more modest elevations in citrulline are detected in argininosuccinate lyase deficiency. Consequently screening will detect "hypomorphic" or "Km variants" of ASS deficiency as well as citrin deficiency [Rinaldo et al., 2004; Schulze et al., 2003]. Several investigators have evaluated the mutation spectrum in patients with mildly elevated citrulline on newborn screening [Haberle et al., 2003]. Some patients, with confirmed mutations in ASS1, have remained asymptomatic for extended periods. This raises the question of what role, if any, therapy has for these patients

From a clinical point of view it would be valuable to distinguish patients with benign disease from those who will require therapy and possibly suffer long term complications. In this series, we reviewed the clinical presentation, genotype and biochemical phenotype of patients diagnosed in our genetics laboratory. Some of these patients were identified by abnormal newborn screening, and others presented with a metabolic crisis. We evaluate the benefit of therapeutic strategies employed in these patients.

\section{MATERIALS AND METHODS}

\section{Patients}

Clinical samples from patients with elevated citrulline were referred to the Medical Genetics laboratories at Baylor College of Medicine, Houston, Texas. Clinical testing was performed as indicated. Research testing was performed with the consent of parents and the assent of the subject as appropriate. Hypomorphic Citrullinemia is defined in a patient with a mean plasma citrulline levels of less than $1,000 \mu \mathrm{M}$ or a peak level of less than $1,500 \mu \mathrm{M}$, with enzymatic or molecular data confirming ASSI as the gene locus. All research procedures were performed in compliance with appropriate federal regulations and with the approval of the Baylor College of Medicine Institutional Review Board.

Enzymology-Argininosuccinate synthetase enzyme assay was performed as previously described [Su et al.,1982] with a reference range of $0.8-3.8 \mathrm{nmol} / \mathrm{min}$ per $\mathrm{mg}$ protein established in our Laboratory over the past 25 years. The lower limit of detection of this assay is $0.02 \mathrm{nmol} / \mathrm{min}$ per $\mathrm{mg}$ protein reflecting approximately $5 \%$ activity. Citrulline incorporation was performed as previously described [Su et al., 1982, Northrup et al., 1990]. Briefly, fibroblasts were incubated in minimal essential media without leucine or arginine but containing ${ }^{14} \mathrm{C}$-citrulline and ${ }^{3} \mathrm{H}$-leucine. After incubation the protein was precipitated 
and the $14 \mathrm{C} / 3 \mathrm{H}$ ratio is determined. If the patient has an enzyme deficiency, the citrulline is not incorporated leading to a reduced ratio.

Sequencing-DNA was extracted from blood, liver, muscle, and cultured skin fibroblasts as available, using a Puregene kit according to the manufacturer's instructions (Gentra Systems Inc., Minneapolis, MN). The entire coding exons and 50 bases of the flanking intron regions of the ASS1 gene were PCR amplified, followed by automated DNA sequencing in both forward and reverse directions using gene specific primers linked to M13 universal sequence primers. The sequencing was performed on an ABI3730XL automated DNA sequencer with Sequencing Analysis Software v5.1 (Applied Biosystems, Foster City, CA, USA). DNA sequences were analyzed using Mutation Surveyor version 2.6.1 (SoftGenetics, State College, PA, USA) and the Genebank sequence: NM_054012 was used as the reference sequence. DNA mutation numbering is based on the cDNA sequence with +1 corresponding to the A of the ATG translation initiation codon. For protein sequence information, the initiation codon is codon 1. In all patients, DNA extraction and sequencing were repeated to ensure correct identification of mutations. Trans-configuration of mutations was confirmed by sequence analysis of parental DNA samples. The detection of homozygous mutations was confirmed by parental DNA analysis. In one case of apparently homozygous mutations where only one parent was available (case 4), and in another where neither parent was available (case 3), the locus was interrogated for intragenic deletions as described elsewhere [Wong et al., 2008].

\section{RESULTS}

A total of 10 patients were identified with hypomorphic citrullinemia. They are summarized in table 1 .

Patient 1 was a 16-year-old female of mixed European ancestry, who presented at 15 years of age with acute liver failure and hyperammonemic encephalopathy. She had a longstanding history of unexplained learning difficulties and behavioral problems. She was 17 weeks pregnant. Plasma amino acids showed a modestly elevated citrulline $(480 \mu \mathrm{M}$, reference range $<42 \mu \mathrm{M}$ ) with an elevated glutamine. She was aggressively treated with intravenous sodium phenylacetate/sodium benzoate along with I.V. arginine. She responded promptly to therapy and was instituted on a protein restricted diet with oral supplementation of arginine and sodium benzoate. Citrulline levels remained less than $500 \mu \mathrm{M}$ during pregnancy. She subsequently gave birth to an unaffected infant who was neurologically normal at 6 months of age. Her subsequent citrulline levels have fluctuated around 1,000$1,500 \mu \mathrm{M}$. Mutation analysis revealed compound heterozygosity for the previously reported c.1168G >A (p.G390R), originally described in a cell line from a patient with classical citrullinemia, [Kobayashi et al., 1990] and a previously unreported mutation c.872A $>\mathrm{C}$ (p.Y291S). It is predicted by Polyphen [Ramensky et al., 2002] that the Y291S change is probably damaging but by SIFT [Ng and Henikoff, 2006] to be tolerated. Polyphen predicts the p.G390R to be possibly damaging and SIFT predicts the change to not be tolerated ASS enzyme activity was deficient in skin fibroblasts $(<0.02 \mathrm{nmol} / \mathrm{min} / \mathrm{mg}$, reference range: $0.8-$ $3.8 \mathrm{nmol} / \mathrm{min}$ per $\mathrm{mg}$ protein). Citrulline incorporation was not performed.

Patient 2 was a 13-year-old female, the only child of nonconsanguineous parents. She presented with mild developmental delay and an episode of decreased consciousness with hyperammonemia $(200 \mu \mathrm{M}$, reference range: $<50 \mu \mathrm{M})$ at 21 months of age. Plasma amino acids showed a modestly elevated citrulline $(200 \mu \mathrm{M})$. ASS activity in skin fibroblasts was indistinguishable from zero $(0.03 \mathrm{nmol} / \mathrm{min} / \mathrm{mg})$. Citrulline incorporation was normal. Mutation testing was not performed. She was treated with sodium benzoate $8 \mathrm{~g} / \mathrm{day}$ in three divided doses and moderate protein restriction. Her citrulline levels remained between 100 
and $200 \mu \mathrm{M}$. This patient continued to have significant neurocognitive difficulties. Her most recent neuropsychiatric testing was performed as part of the urea cycle rare disease clinical research network (RDCRN) longitudinal study. It revealed defects in attention and independence with a full scale IQ of approximately 50. She also suffered from seizures and disabling tremors.

Patient 3 was an 8-year-old male with a family history of significant psychological problems in his biological mother and possible consanguinity. He presented at 3 years of age for evaluation of mild global developmental delay. Laboratory investigation revealed a significantly elevated ammonia $(170 \mu \mathrm{M}$, normal range $<50 \mu \mathrm{M})$. His citrulline levels have fluctuated from $200-400 \mu \mathrm{M}$ over the following years. Dietary protein restriction was initiated at the time of diagnosis. This resulted in a significant improvement in school performance and he is maintained on his protein recommended daily allowance [National Academy of Science, 2002/2005]. He has maintained grade appropriate level in a mainstream school. DNA testing revealed a previously unreported homozygous c.216C $>\mathrm{G}$ (p.F72L) mutation. Polyphen analysis predicts this variant to be benign but SIFT predicts that it is not tolerated. Enzyme analysis and citrulline incorporation have not been performed to confirm the pathogenicity of this variant. Sequencing of SLC25A13 (Citrin) was normal. Parental DNA was not available for analysis; however no deletions were detected at either locus.

Patient 4 was a 2-year-old male. He was born at term to parents of Mexican ancestry who are first cousins. Newborn screening revealed an isolated elevated citrulline $(192 \mu \mathrm{M}$, reference range $<35 \mu \mathrm{M}$ ) with no argininosuccinic acid on Wisconsin expanded newborn screening. With a presumptive diagnosis of citrullinemia, the patient was started on a low protein diet and arginine supplementation. Citrulline levels have ranged between (548-986 $\mu \mathrm{M})$, suggestive of mild citrullinemia. The patient has never had an episode of hyperammonemia. Enzyme analysis revealed activity at the lower limit of normal $(0.8 \mathrm{nmol} /$ $\mathrm{min} / \mathrm{mg}$ ) with normal citrulline incorporation. Mutation analysis of the ASS1 gene demonstrated a homozygous, previously reported c.535T $>\mathrm{C}$ (p.W179R) mutation [Haberle et al., 2003]. SIFT predicts that this mutation is not tolerated [Ng and Henikoff, 2006] and Polyphen concludes that it is probably damaging. Sequencing of SLC25A13 (Citrin) was normal. Paternal DNA was not available for analysis, however no deletions were detected at either locus. Given the patient's mild phenotype, low normal ASS activity, and the mild metabolic phenotype of previously ascertained patients with the same mutation, dietary restriction was relaxed. The patient remained clinically well with normal growth and development.

Patient 5 was a 1-year-old male whose parents originate from two different parts of India. He presented with an elevated citrulline of $1000 \mu \mathrm{M}$ on expanded newborn screening. He had been modestly protein restricted and had normal development. His citrulline levels have fluctuated between 350 and $1000 \mu \mathrm{M}$. Mutation analysis of the ASS1 gene demonstrated a heterozygous, previously described mutation c.1168G>A (p.G390R) [Kobayashi et al., 1990]. A heterozygous, previously unreported missense variant c.815G>A (p.R272H) was also found. This missense mutation is at the same codon as a previously reported mutation (p.R272C) and is predicted by Polyphen to be possibly damaging and by SIFT to not be tolerated. Enzyme analysis and citrulline incorporation have not been performed.

Patient 6 was a 10-month-old asymptomatic male who presented with an elevated citrulline on Texas expanded newborn screening ( $24 \mathrm{hr}$ citrulline $382 \mu \mathrm{M}$, 2nd screen $911 \mu \mathrm{M}$, upper limit of normal 180, reflex plasma amino acids 981 at 11 days of age). He was modestly protein restricted with dietary supplementation of Cyclinex. He had normal development. Mutation analysis of the ASS1 gene demonstrated a heterozygous c.535T>C (p.W179R) and 
heterozygous c.853C $>\mathrm{T}$ (p.R279X) mutation in this patient. Enzyme analysis and citrulline incorporation had not been performed.

Patient 7 was a 1-year-old asymptomatic child of nonconsanguineous parents from India. She presented with an isolated elevated citrulline on newborn screening. She was modestly protein restricted and had no significant neurocognitive difficulties. Mutation analysis revealed a previously unreported homozygous mutation c.262C $>\mathrm{G}$ (p.L88I). SIFT predicts this to not be tolerated, but Polyphen predicts that it is probably benign. Enzyme analysis for ASS was deficient on skin fibroblasts $(<0.02 \mathrm{nmol} / \mathrm{min} / \mathrm{mg})$ confirming the deleterious nature of this mutation. Citrulline incorporation was not performed.

Patient 8 was a 1-year-old Caucasian female who presented with a "slightly elevated" citrulline on expanded newborn screening (Pediatrix, now StepOne newborn screening). Reflex plasma amino acids revealed a citrulline of $236 \mu \mathrm{M}$ at 2 weeks of age. She remained asymptomatic with normal glutamine and no hyperammonemia on a modestly protein restricted diet. Enzyme analysis for ASS was low on skin fibroblasts $(0.4 \mathrm{nmol} / \mathrm{min} / \mathrm{mg})$. Citrulline incorporation and gene sequencing was not performed.

Patient 9 was a 4-year-old male who presented with an elevated citrulline on Wisconsin newborn screening ( $185 \mu \mathrm{M}$ at 48 hours; $385 \mu \mathrm{M}$ at 6 days of age (cutoff $85 \mu \mathrm{M})$ ). ASS enzyme assay performed in Amsterdam revealed reduced activity (5u, control range 26+l $-9 \mathrm{u}$ ), citrulline incorporation was not performed. Mutation analysis performed at UKM, Munster, Germany showed compound heterozygosity for two previously described mutations. The p.V263M mutation has been described in mildly affected patients [Harble et al., 2003] whereas the p.G324S is associated with classical citrullinemia [Kobayashi et al., 1990]. Polyphen predicts the p.V263M variant to be benign and the p.G324S to be probably damaging but SIFT predicts both mutations would not be tolerated. He remained cognitively intact and asymptomatic on his recommended daily allowance of protein, [National Academy of Science, 2002/2005] with $100 \mathrm{mg} / \mathrm{k} / \mathrm{d}$ of arginine supplementation.

Patient 10 was a 6-month-old female male who presented with an elevated citrulline on Wisconsin newborn screening ( $101 \mu \mathrm{M}$ at 48 hours; $109 \mu \mathrm{M}$ at 7 days of age (cutoff 85 $\mu \mathrm{M})$ ). Reflex plasma amino acids indicated an isolated elevated citrulline of $185 \mu \mathrm{M}$ Sequencing showed compound heterozygosity for p.Q40L and p.S180N. The p.Q40L mutation has previously been reported in patients with hypomorphic or mild citrullinemia [Haberle et al., 2003] whereas the p.S180N has been reported in patients with classical citrullinemia [Kobyashi et al., 1990]. SIFT predicts both of these mutations to not be tolerated. Polyphen predicts both the p.Q40L and the p.S180N mutations to be probably damaging. The patient remained asymptomatic on her recommended daily allowance of protein $(1.8 \mathrm{gram} / \mathrm{kg} / \mathrm{d}$, [National Academy of Science 2002/2005]) with $100 \mathrm{mg} / \mathrm{k} / \mathrm{d}$ of arginine supplementation.

\section{DISCUSSION}

Distinction of primary ASS deficiency from citrin deficiency in patients presenting with modestly elevated citrulline levels on newborn screening is essential because the treatment for these conditions is divergent. As citrin deficiency is associated with a normal ASS activity in skin fibroblasts [Kobayashi et al., 1993], we hypothesized that enzymatic testing may allow segregation of these conditions. Although three of the six patients we could evaluate had deficient ASS activity and two others had activity below the normal range, one had normal activity. Therefore ASS enzyme testing, in isolation, cannot be used to exclude mutations in ASS1 as the cause of elevated citrulline. However, since both symptomatic patients, with enzyme data available, were deficient and the patients with residual activity 
have, to date, remained asymptomatic, normal ASS enzyme activity may be indicate a good prognosis. Conversely, with one asymptomatic patient with no detectable activity, the absence of activity does not mandate a poor outcome. However, with such a limited number of patients this pattern needs to be confirmed in other patients.

Our lab has previously shown in one patient a " $\mathrm{Km}$ " variant in trans with a null variant is sufficient to lead to detectable but reduced citrulline incorporation [Kobayashi et al., 1991] Additionally, those with 5 to $10 \%$ residual activity have normal citrulline incorporation [Su et al., 1982]. In these cases with less elevated citrulline, we have been able to show normal citrulline incorporation. This suggests that citrulline incorporation might be a useful clinical test for distinguishing hypomorphic patients from those with a more severe deficiency but will require further evaluation.

Our sequence data is consistent with another large series, [Haberle et al., 2003] in that the c. 535T $>\mathrm{C}$ (p.W179R), is the most common mutation in our patients (three of the 16 alleles), but previously undescribed missense changes are more prevalent. In addition to the difficulty created by large numbers of undescribed mutations, we see a significant discrepancy between the predictions of two commonly used in-silico tools with enzymatic data and each other. Neither of these tools usefully distinguish hypomorphic mutations from classical mutations. This difficulty in predication is consistent with other disease loci [Tchernitchko et al., 2004] and suggests that enzymatic evaluation is necessary, especially in the context of undescribed mutations.

The prompt response to dietary therapy in Patient 3 is encouraging and suggests that a modestly protein restricted diet should be considered in all such patients to ensure optimal development. However, it is possible that not all children with this condition require dietary restriction as a good neurocognitive development was previously described in three of four patients in two families with the p.W179R mutation without dietary restriction [Haberle et al., 2003]. Although it is not clear if Patient 2's poor long-term outcome is as a result of damage sustained prior to diagnosis, we demonstrate that even with optimal dietary management subsequent to diagnosis, not all children will have a good long-term neurological outcome, and therefore prognosis must be guarded.

The documentation of a normal pregnancy outcome in a patient presenting in pregnancy is encouraging for the long term reproductive options of this class of patients and is consistent with the recently documented report of 2 pregnancies in patients with ASS deficiency [Enns et al., 2005; Potter et al., 2004]. Interestingly, one of these patients with IVS15-1G > C and p.K310Q, mutations in ASS1, had deficient ASS activity and citrulline incorporation in our laboratory suggesting a more severe deficiency. However these patients' outcomes are in marked contrast to the three previously reported patients diagnosed in pregnancy with ASS deficiency. One patient with p.R86H and p.G390R mutations developed fulminant hepatic failure 8 months into pregnancy, underwent a pregnancy termination and died 5 months later. The other two patients also had significant adverse effects in pregnancy [Keiko Kobayashi, personal correspondence] [Ito et al., 2004]. What is unclear, at this point, is why pregnancy seems to precipitate such crisis. It can be hypothesized that these patients have sufficient residual ASS activity for normal clinical functioning but the increased metabolic stress of early pregnancy, or more likely mild pregnancy associated liver disease, tip the patients below the critical minimal function to maintain adequate functioning.

To our knowledge this is the first reported use of nitrogen scavenging drugs (sodium phenylacetate/sodium benzoate) in pregnancy. The prompt response to therapy and the good long-term outcome of both mother and child is encouraging. However, more studies will be required before this therapy can be confidently recommended in pregnancy 
In conclusion residual ASS enzyme activity on fibroblasts may be normal in children with hypomorphic citrullinemia. We therefore recommend clinical sequencing of ASS1 and SLC25A13 in patients presenting with elevated citrulline on newborn screening. ASS enzyme activity may be a useful tool in predicting long term outcome and is necessary for interpreting functional effects of sequence variants. It therefore continues to have a role in the diagnosis of this disorder. Protein restriction and continued monitoring is warranted to prevent neurocognitive dysfunction

\section{Acknowledgments}

The authors wish to thank Jacqueline Heidorn for assistance with the ASS enzyme assay and citrulline incorporation and the urea cycle RDCRN for allowing us to use the neurocognitive data on Patient 2. This study was supported in part by an NIH career development award to DD (K12 RR17665)

\section{References}

Bachmann C. Outcome and survival of 88 patients with urea cycle disorders: a retrospective evaluation. Eur J Pediatr. 2003; 162:410-416. [PubMed: 12684900]

Beaudet AL, O'Brien WE, Bock HG, Freytag SO, Su TS. The human argininosuccinate synthetase locus and citrullinemia. Adv Hum Genet. 1986; 15:161-196. 291-292. [PubMed: 3513483]

Enns GM, O’Brien WE, Kobayashi K, Shinzawa H, Pellegrino JE. Postpartum "psychosis" in mild argininosuccinate synthetase deficiency. Obstet Gynecol. 2005; 105(5 Pt 2):1244-1246. [PubMed: 15863597]

Haberle J, Pauli S, Schmidt E, Schulze-Eilfing B, Berning C, Koch HG. Mild citrullinemia in Caucasians is an allelic variant of argininosuccinate synthetase deficiency (citrullinemia type 1). Mol Genet Metab. 2003; 80:302-306. [PubMed: 14680976]

Ito S, Kurasawa G, Yamamoto K, Furuta I, Ishihara F, Kobayashi K, Saheki T, Matsuura T, Yamauchi M, Kakinoki H. A pregnant patient with fulminant hepatic failure was found to carry a novel missense mutation in the argininosuccinate synthetase gene. J Gastroenterol. 2004; 39:1115-1117. [PubMed: 15580409]

Kobayashi K, Jackson MJ, Tick DB, O’Brien WE, Beaudet AL. Heterogeneity of mutations in argininosuccinate synthetase causing human citrullinemia. J Biol Chem. 1990; 265:11361-11367. [PubMed: 2358466]

Kobayashi K, Rosenbloom C, Beaudet AL, O'Brien WE. Additional mutations in argininosuccinate synthetase causing citrullinemia. Mol Biol Med. 1991; 8:95-100. [PubMed: 1943692]

Kobayashi K, Shaheen N, Kumashiro R, Tanikawa K, O’Brien WE, Beaudet AL, Saheki T. A search for the primary abnormality in adult-onset type II citrullinemia. Am J Hum Genet. 1993; 53:10241030. [PubMed: 8105687]

McMurray WC, Mohyuddin F, Rossiter RJ, Rathbun JC, Valentine GH, Koegler SJ, Zarfas DE. Citrullinuria: a new aminoaciduria associated with mental retardation. Lancet. 1962; I:138.

National Academy of Science: Food and Nutrition Board. Dietary Reference Intakes: Energy, Carbohydrates, Fiber, Fat, Fatty Acids, Cholesterol, Protein, and Amino Acids. National Academies Press; Washington, DC: 2002/2005.

$\mathrm{Ng}$ PC, Henikoff S. Predicting the effects of amino acid substitutions on protein function. Annu Rev Genomics Hum Genet. 2006; 7:61-80. [PubMed: 16824020]

Northrup H, Beaudet AL, O'Brien WE. Prenatal diagnosis of citrullinaemia: review of a 10-year experience including recent use of DNA analysis. Prenat Diagn. 1990; 10:771-779. [PubMed: 2075179]

Potter MA, Zeesman S, Brennan B, Kobayashi K, Gao HZ, Tabata A, Saheki T, Whelan DT. Pregnancy in a healthy woman with untreated citrullinemia. Am J Med Genet Part A. 2004; 129A: 77-82. [PubMed: 15266621]

Ramensky V, Bork P, Sunyaev S. Human non-synonymous SNPs: server and survey. Nucleic Acids Res. 2002; 30:3894-3900. [PubMed: 12202775] 
Rinaldo P, Tortorelli S, Matern D. Recent developments and new applications of tandem mass spectrometry in newborn screening. Curr Opin Pediatr. 2004; 16:427-433. [PubMed: 15273505]

Ruitenbeek W, Kobayashi K, Iijima M, Smeitink JA, Engelke UF, De Abreu RA, Kwast HT, Saheki T, Boelen CA, De Jong JG, Wevers RA. Moderate citrullinaemia without hyperammonaemia in a child with mutated and deficient argininosuccinate synthetase. Ann Clin Biochem. 2003; 40(Pt 1): 102-107. [PubMed: 12542919]

Schulze A, Lindner M, Kohlmuller D, Olgemoller K, Mayatepek E, Hoffmann GF. Expanded newborn screening for inborn errors of metabolism by electrospray ionization-tandem mass spectrometry: results, outcome, and implications. Pediatrics. 2003; 111(6 Pt 1):1399-1406. [PubMed: 12777559]

Su TS, Bock HG, Beaudet AL, O'Brien WE. Molecular analysis of argininosuccinate synthetase deficiency in human fibroblasts. J Clin Invest. 1982; 70:1334-1339. [PubMed: 7174798]

Tchernitchko D, Goossens M, Wajcman H. In silico prediction of the deleterious effect of a mutation: proceed with caution in clinical genetics. Clin Chem. 2004; 50:1974-1978. [PubMed: 15502081]

Wick H, Bachmann C, Baumgartner R, Brechbuhler T, Colombo JP, Wiesmann U, Mihatsch MJ, Ohnacker H. Variants of citrullinaemia. Arch Dis Child. 1973; 48:636-641. [PubMed: 4783005]

Wong LJ, Dimmock D, Geraghty MT, Quan R, Lichter-Konecki U, Wang J, Brundage EK, Scaglia F, Chinault AC. Utility of Oligonucleotide Array-Based Comparative Genomic Hybridization for Detection of Target Gene Deletions. Clin Chem. 2008 


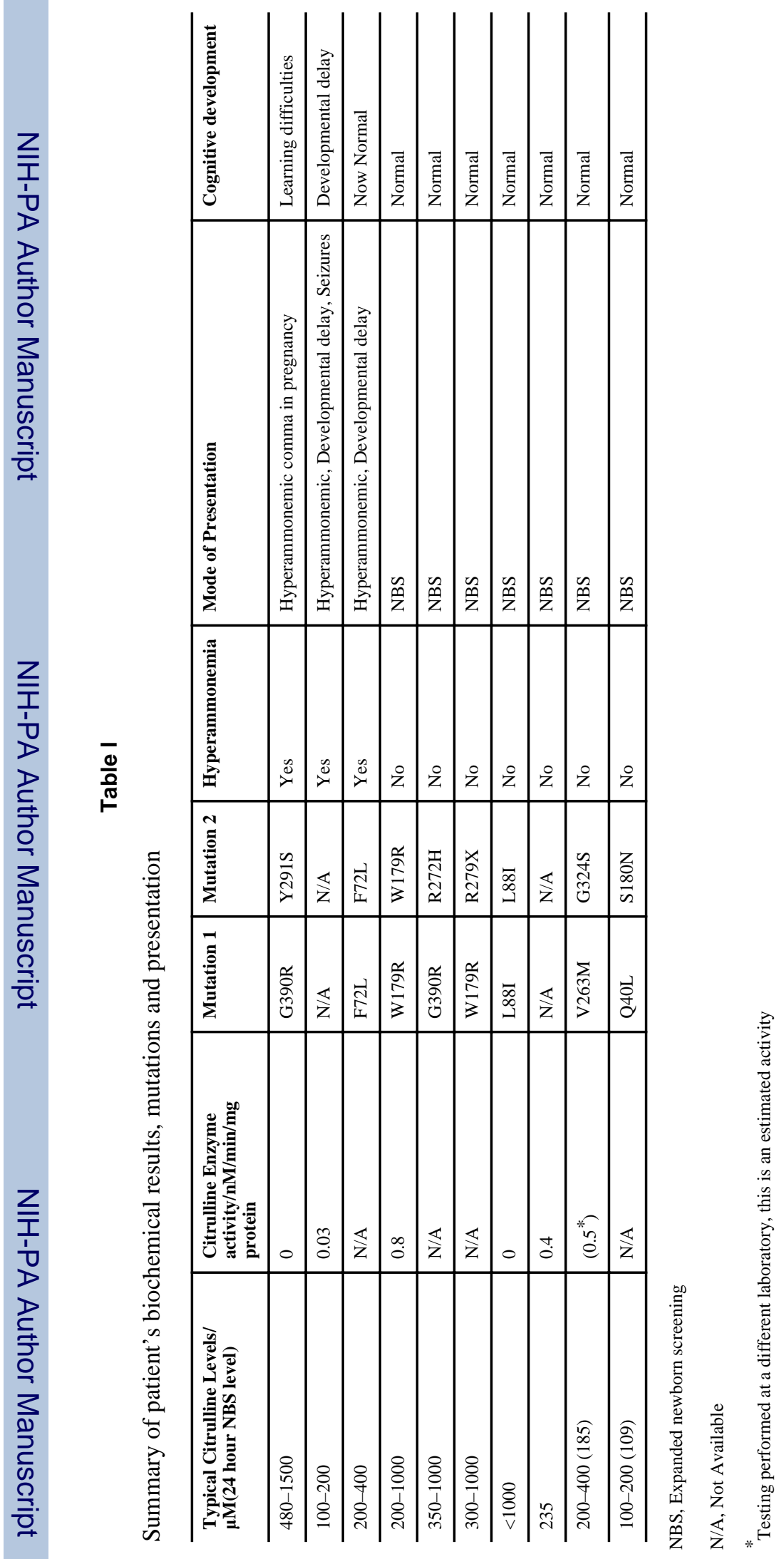

